\title{
Structure prediction for multicomponent materials using biminima
}

\author{
D. Schebarchov ${ }^{1, *}$ and D. J. Wales ${ }^{1, \dagger}$ \\ ${ }^{1}$ University Chemical Laboratories, Lensfield Road, Cambridge CB2 1EW, UK
}

(Dated: October 15, 2014)

\begin{abstract}
The potential energy surface of a heteroparticle system will contain points that are local minima in both coordinate space and permutation space for the different species. We introduce the term biminima to describe these special points, and we formulate a deterministic scheme for finding them. Our search algorithm generates a converging sequence of particle-identity swaps, each accompanied by a number of local geometry relaxations. For selected binary atomic clusters of size $N=N_{A}+$ $N_{B} \leq 98$, convergence to a biminimum on average takes $3 N_{A} N_{B}$ relaxations, and the number of biminima grows with the preference for mixing. The new framework unifies continuous and combinatorial optimization, providing a powerful tool for structure prediction and rational design of multicomponent materials.
\end{abstract}

PACS numbers: 68.65.-k, 61.46.-w, 61.66.Dk

Introduction. A recurrent goal in computational materials science is efficient and reliable structure prediction in atomic [1, 2], molecular [3], and mesoscopic systems [4]. This objective is often framed as a global optimisation problem [5], whose solution is the configuration with the lowest energy. Finding the global minimum and other low-lying structures is generally non-trivial [5-11], and for multicomponent systems the task is exacerbated by permutations of inequivalent constituents [12, 13]. The extra layer of complexity also endows the energy function with various permutational properties, which we exploit in the present work to construct a general framework for global optimisation that is precise and efficient in the combinatorial sense.

Most common strategies for global optimisation operate by sampling local minima [6-11]. The search procedure usually comprises (i) a local geometry minimisation step and (ii) a move set, with the two ingredients iterated in tandem. The main distinction between variants of basin-hopping [6-8], "Lamarckian" genetic algorithms $[9,10]$, and other strategies [11] is in the choice of moves and how they are used to sample local minima. The local minimisation step provides a many-to-one mapping [6] $f_{1}: \mathcal{S} \mapsto \mathcal{S}^{\prime}$, where $\mathcal{S}$ represents the entire configuration space and $\mathcal{S}^{\prime} \subseteq \mathcal{S}$ is the set of distinct local minima. Systematic use of $f_{1}$ is a highly effective means of reducing the sampling domain, and improving the search efficiency. It also alters the occupation probability of each minimum in a manner that can facilitate global optimisation for multiple-funnel energy landscapes [5, 14].

For multicomponent systems, broken permutational symmetry will enlarge $\left|\mathcal{S}^{\prime}\right|$ by a combinatorial factor [12, 13]. This formidable complication can be mitigated (with limited success [15]) by including a random particleidentity swap in the set of escape moves $[8,10]$. Another strategy that has proven more effective is to execute a sequence of swaps until a loose termination criterion is fulfilled [16]. Here we exploit the latter approach by deriving a framework based on a precise convergence crite- rion. In short, we propose a deterministic search for configurations in $\mathcal{S}^{\prime}$ that define local minima in the permutation space of particle-identity swaps [17]. The method can also be described as a mapping, $f_{2}: \mathcal{S} \mapsto \mathcal{S}^{\prime \prime}$, where $\mathcal{S}^{\prime \prime} \subseteq \mathcal{S}^{\prime}$ contains the biminima. Note that identity swaps are no longer an escape move, but a means of extending the conventional notion of local minimisation to endow it with a hybrid continuous-permutational meaning. We now present a detailed description of this framework and apply it to binary atomic clusters.

Generalities. We are concerned with a system of $N=$ $\sum_{\alpha=1}^{M} N_{\alpha}$ particles, where $M$ is the number of distinct species and each $N_{\alpha}$ is fixed. Each particle has a unique index $i$, a label $l_{i} \in\{\alpha\}_{\alpha=1}^{M}$, and coordinates $\mathbf{x}_{i} \in \Omega \subset$ $\mathbb{R}^{d}$, where $\Omega$ is a bounded $d$-dimensional region. The list of particle indices, $\mathcal{I} \equiv\{i\}_{i=1}^{N}$, is partitioned by the labels: $\mathcal{I}=\cup_{\alpha=1}^{M} \mathcal{I}_{\alpha}$, where $\mathcal{I}_{\alpha} \equiv\left\{i \in \mathcal{I}: l_{i}=\alpha\right\}$.

The configuration energy of the system is modelled by a scalar function $E(\mathbf{X}, \mathcal{L} ; \mathcal{P})>-\infty$, where $\mathbf{X} \equiv\left\{\mathbf{x}_{i}\right\}_{i=1}^{N}$, $\mathcal{L} \equiv\left\{l_{i}\right\}_{i=1}^{N}$, and $\mathcal{P} \equiv\left\{p_{k}\left(\mathcal{L}_{k}\right)\right\}_{k=1}^{K}$ is a set of $K$ parameters whose values may depend on a subset $\mathcal{L}_{k} \subseteq \mathcal{L}$. As a simple illustrative example consider a Lennard-Jones potential:

$$
E_{L J}=4 \sum_{i=1}^{N-1} \sum_{j=i+1}^{N} \epsilon_{l_{i} l_{j}}\left[\left(\frac{\sigma_{l_{i} l_{j}}}{r_{i j}}\right)^{12}-\left(\frac{\sigma_{l_{i} l_{j}}}{r_{i j}}\right)^{6}\right],
$$

where $r_{i j}=\left|\mathbf{x}_{i}-\mathbf{x}_{j}\right|$ is the Euclidean distance between atoms $i$ and $j$, and the parameters $\epsilon_{l_{i} l_{j}}$ (the well depth) and $\sigma_{l_{i} l_{j}}$ (atomic radius) can take different values for each of the $M(M-1) / 2$ distinct combinations of $l_{i} l_{j}$. $E(\mathbf{X}, \mathcal{L} ; \mathcal{P})$ can change value when two distinct labels are swapped, and a sequence of swaps could generate up to $N ! / \prod_{\alpha} N_{\alpha}$ ! distinct configurations.

We seek configurations with the lowest value of $E(\mathbf{X}, \mathcal{L} ; \mathcal{P})$ in a search space $\mathcal{S}=\{\mathbf{X}\}$ that depends on $d, N$ and $\Omega$. Following standard practice we focus on the subset of local minima, $\mathcal{S}^{\prime}$, where formally $\mathbf{X}^{\prime} \in \mathcal{S}^{\prime}$ if (and only if) there exists $\delta>0$ such that $E(\mathbf{X}, \mathcal{L} ; \mathcal{P}) \geq$ 


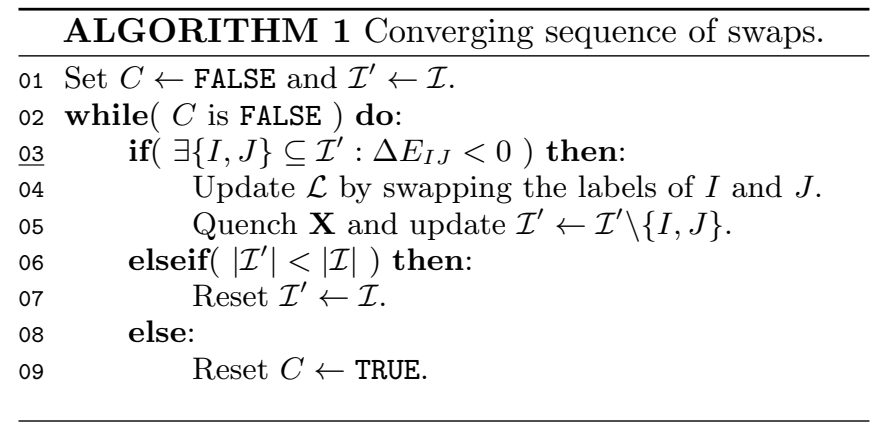

$E\left(\mathbf{X}^{\prime}, \mathcal{L} ; \mathcal{P}\right)$ for all $\mathbf{X} \in \mathcal{S}$ with $\left|\mathbf{X}-\mathbf{X}^{\prime}\right|<\delta$. To help us narrow the candidate set we introduce the swap-gain matrix, denoted by $\Delta E_{i j}\left(\mathbf{X}^{\prime}, \mathcal{L} ; \mathcal{P}\right)$, which quantifies the change in energy due to a label swap, $l_{i} \leftrightarrow l_{j}$, followed by a local relaxation of $\mathbf{X}$ (i.e. a quench). Note that swapping two labels is the "smallest" permutation that does not alter the stoichiometry, while quenching after the swap is necessary to ensure that we remain in $\mathcal{S}^{\prime}$. It is also worth stressing that $\Delta E_{i j}$ can be computed for any $E(\mathbf{X}, \mathcal{L} ; \mathcal{P})$. We now define a locally optimal label assignment to be one that satisfies

$$
\Delta E_{i j} \geq 0, \quad \forall i, j \in \mathcal{I} .
$$

That is, (2) requires all the elements of the symmetric matrix $\Delta E_{i j}$ to be non-negative. The set $\left(\mathcal{S}^{\prime \prime}\right)$ of configurations satisfying this criterion defines the image of the many-to-one mapping $f_{2}: \mathcal{S} \mapsto \mathcal{S}^{\prime \prime}$.

The matrix $\Delta E_{i j}$ can be arranged into an $M \times M$ block form, where each block contains $N_{\alpha} \times N_{\beta}$ elements for all the $\alpha \beta$-type swaps. Since $\Delta E_{i j}=\Delta E_{j i}$ and blocks with $\alpha=\beta$ are immaterial, only $M(M-1) / 2$ blocks with a total of $\frac{1}{2} \sum_{\alpha} \sum_{\beta \neq \alpha} N_{\alpha} N_{\beta}$ elements need be computed when satisfying (2), and each block can be treated separately. (For instance, one can sweep the different blocks in a fixed sequence and iterate until all of the individual blocks have converged, but there may be a more efficient way.) Hence, without loss of generality, we now consider a single $\alpha \beta$ block with $\alpha \neq \beta$, corresponding to the simplest non-trivial case.

Methods. We implement the mapping $f_{2}: \mathcal{S} \mapsto \mathcal{S}^{\prime \prime}$ by adapting standard graph partitioning techniques [18], which in the present context aim to minimise the energy by interchanging particle identities. A key difference is that now each swap is accompanied by a quench. Our algorithm (ALG. 1) consists of a single loop, which iterates until the logical variable $C$ switches from the initial value of FALSE to TRUE. The switch will happen only when (2) is satisfied. Note the use of an auxiliary list $\mathcal{I}^{\prime} \subseteq \mathcal{I}$, from which we incrementally remove the indices $I$ and $J$ that have been selected for a swap. The list is fully replenished when $\Delta E_{i j} \geq 0, \forall i, j \in \mathcal{I}^{\prime}$. This formulation reduces the number of candidates at each successive iteration of the loop, improving the net efficiency and imposing a "memory" effect. In contrast to our previous study [17], we

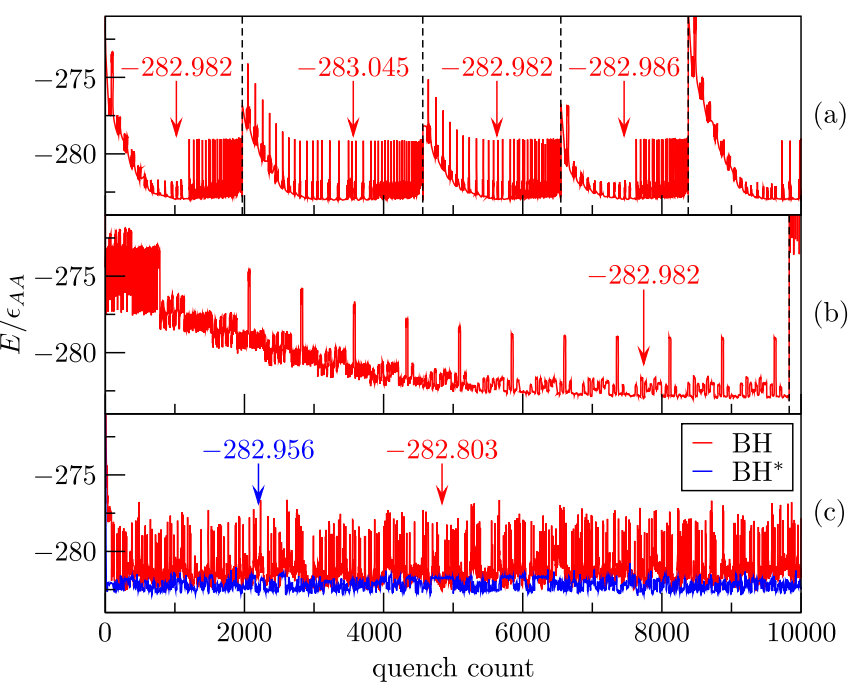

FIG. 1. Quenched configuration energy versus quench count for (a) fast and (b) slow biminimisation schemes as well as (c) two variants of basin-hopping. The initial configuration is the same in all cases. In (a) and (b), the dashed lines mark a random permutation, while the numbers with a downwardpointing arrow indicate the energy of each biminimum and the quench where it is first encountered. In (c) we indicate only the lowest energy for each dataset.

reject swaps with $\Delta E_{i j} \geq 0$ to avoid climbing "uphill".

The computational bottleneck in ALG. 1 is on the third line, requiring either $\{I, J\} \subseteq \mathcal{I}^{\prime}$ with $\Delta E_{I J}<0$ or a verification that no such $\{I, J\}$ exists. To fulfil this requirement we construct an iterative procedure designed to converge on a relatively low (and often the lowest) swap gain within one $\alpha \beta$ block. The procedure involves evaluating rows and columns of the block in an alternating sequence (see below), until a row and column intersecting at a particular $\Delta E_{i^{\prime} j^{\prime}}$ are found. If $\Delta E_{i^{\prime} j^{\prime}}<0$, we set $\{I, J\}=\left\{i^{\prime}, j^{\prime}\right\}$ and terminated the procedure. Otherwise, the $\alpha \beta$ block is reduced to hitherto unevaluated rows/columns, and a new sequence is initiated on this smaller sub-block. Failure to locate $\Delta E_{i j}<0$ before all the rows/columns are depleted will mean that (2) is satisfied for the entire $\alpha \beta$ block.

To describe the alternating sequence, we shall denote it by $\left(i_{k}\right)_{k=1}^{k_{c}}$, where $i_{k} \in \mathcal{I}_{\alpha} \cup \mathcal{I}_{\beta}$ and $k_{c}<\infty$ is defined below. We first choose $i_{1}$ by the lowest flip gain [17], then increment $k$ in steps of one and select $i_{k}$ to minimise $\Delta E_{i_{k} i_{k-1}}$. The sequence alternates between $\alpha$ - and $\beta$-type particles by construction, and it is expected to eventually attain a period of two. That is, $i_{k}=i_{k-2}$ for all $k>k_{c}>2$, where $k_{c}$ marks the start of the periodic behaviour. The periodicity is expected when $\Delta E_{i_{k} i_{k-1}}$ is the lowest in column/row $i_{k}$ and row/column $i_{k-1}$ of the $\alpha \beta$ (sub-)block. The lowest swap gain in the (sub-)block will always satisfy this criterion, but there may be others that also qualify.

Results. For our case study we consider binary 


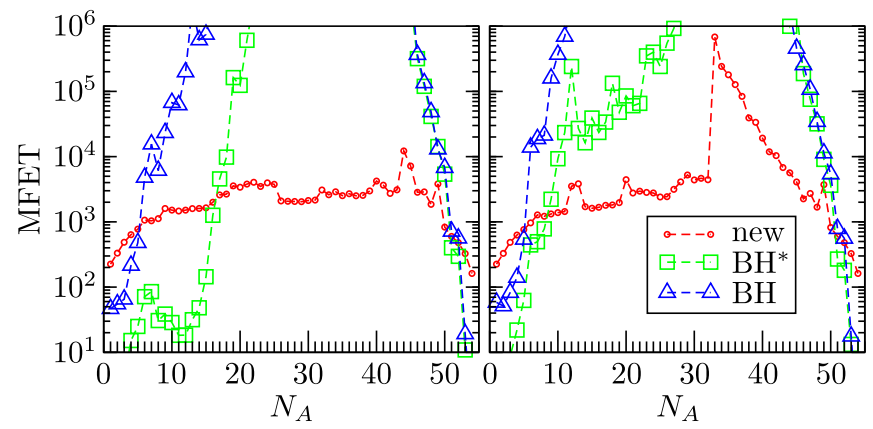

FIG. 2. Mean first-encounter times, measured in number of quenches, from a hundred independent searches for $\mathrm{BLJ}_{55}$ icosahedra with particle-size mismatches of $5 \%$ (left) and $10 \%$ (right) and all stoichiometries. All three search methods were targeting the same energy for each $N_{A}$.

Lennard-Jones clusters $\left(\mathrm{BLJ}_{N}\right)$ of size $N=N_{A}+N_{B}$ and $E(\mathbf{X}, \mathcal{L} ; \mathcal{P})$ defined by $(1)$. To keep the number of parameters small and retain a rich variety of structures [19], we set $\epsilon_{A A}=\epsilon_{A B}=\epsilon_{B B}$ and fix $\sigma_{A B}$ by the Lorentz rule: $\sigma_{A B}=\left(\sigma_{A A}+\sigma_{B B}\right) / 2$. Taking $\sigma_{A A}$ as a unit of length leaves just three parameters: $N, N_{A}$ and $\sigma_{B B} / \sigma_{A A}$.

As a first test case we choose $N=55, N_{A}=28$ and $\sigma_{B B} / \sigma_{A A}=1.1$, which correspond to sizes, compositions and particle size mismatches commonly encountered in nanoalloys $[1,13]$. To generate a starting configuration we first prearrange $\mathbf{X}$ into the ground-state geometry for $\mathrm{LJ}_{55}$ (closed-shell icosahedron). We then randomly choose $\mathcal{L}$ (while respecting the stoichiometry) and quench $\mathbf{X}$. The prepared structure is then evolved using the deterministic scheme for $10^{4}$ quenches, using another random choice of $\mathcal{L}$ and a quench to escape from a converged biminimum. The configuration energy during this evolution is shown in Fig. 1a, exhibiting a gradual downward trend after each escape and spikes at shorter intervals due to unfavourable swaps. To show the effects of (i) $\mathcal{I}^{\prime}$ in ALG. 1 and (ii) our iterative approach to scanning the matrix $\Delta E_{i j}$, we also use a simpler scheme where all of the non-zero matrix elements are evaluated on every swap, which guarantees that the best possible swap is executed each time. The result is plotted in Fig. 1b, showing a slower convergence to the first biminimum compared to Fig. 1a.

In Fig. 1c we provide results for conventional [6] and self-guided [17] basin-hopping (BH) algorithms with random exchange moves. For conventional $\mathrm{BH}$ the temperature was adjusted every fifty quenches to maintain a mean acceptance ratio of 0.5 , yielding an average temperature of 0.2 . For the self-guided variant $\left(\mathrm{BH}^{*}\right)$ the temperature was kept fixed at 0.2 . The lowest energy from $10^{4}$ quenches is indicated for both variants, with neither of them beating any of the biminima in Fig. 1a. Hence, repeated biminimisation is more effective at finding the lowest-lying permutational isomers within a particular

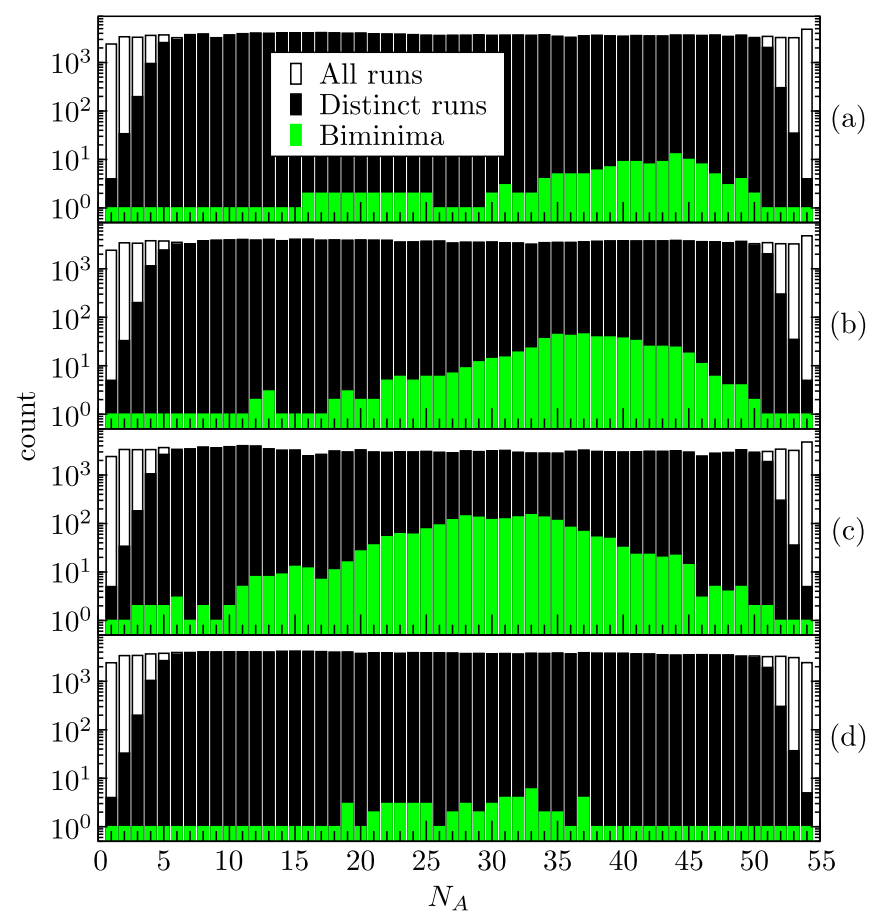

FIG. 3. Counts of distinct biminima from independent runs for $\mathrm{BLJ}_{55}$ with closed-shell icosahedral geometry and: $\sigma_{B B} / \sigma_{A A}=1.05$ in (a) and 1.10 in (b)-(d); $\epsilon_{B B} / \epsilon_{A A}=1.0$ in (a)-(b) and 0.5 in (c)-(d); $\epsilon_{A B} / \epsilon_{A A}=1.0$ in (a)-(b), 1.2 in (c) and 0.7 in $(d)$.

geometric motif (i.e. homotops [12]), and the mean firstencounter times (MFETs) plotted in Fig. 2 further confirm this conclusion. Even without statistically reliable MFETs $>10^{6}$, it is clear that repeated biminimisation can outperform both variants of $\mathrm{BH}$ by orders of magnitude when $\min \left(N_{A}, N_{B}\right) \gtrsim 5$.

In Fig. 2 we compare MFETs for BLJ $\mathrm{J}_{55}$ closed-shell icosahedra with $5 \%$ and $10 \%$ size mismatch. For $10 \%$ mismatch biminimisation exhibits a striking increase in MFET at $N_{A}=33$, which we link to a narrow catchment basin in permutation space. We can estimate (by counting) that the probability of convergence to the lowestlying biminimum is $\sim 0.005$, while for the second-lowest it is $\sim 0.5$. In contrast, for $N_{A}=32$ the occupation probability of the lowest-lying biminimum is $\sim 0.5$, and for the second-lowest it is $\sim 0.03$. This simple analysis hints at the role of the underlying sublandscape topography in permutation space, which can be characterised by analogy with energy landscapes in coordinate space [5].

Note that $E(\mathbf{X}, \mathcal{L} ; \mathcal{P})$ comprises a family of sublandscapes, with each member defined by a projection of $E(\mathbf{X}, \mathcal{L} ; \mathcal{P})$ onto the subspace spanned by permutations of $\mathcal{L}$ at fixed $\mathbf{X}$. Our policy of quenching $\mathbf{X}$ after every permutation transforms the projection each time, giving rise to an effective sublandscape that may not necessarily be singe valued. That is, the same $\mathcal{L}$ may be mapped to different values of $E(\mathbf{X}, \mathcal{L} ; \mathcal{P})$ during a sequence of 


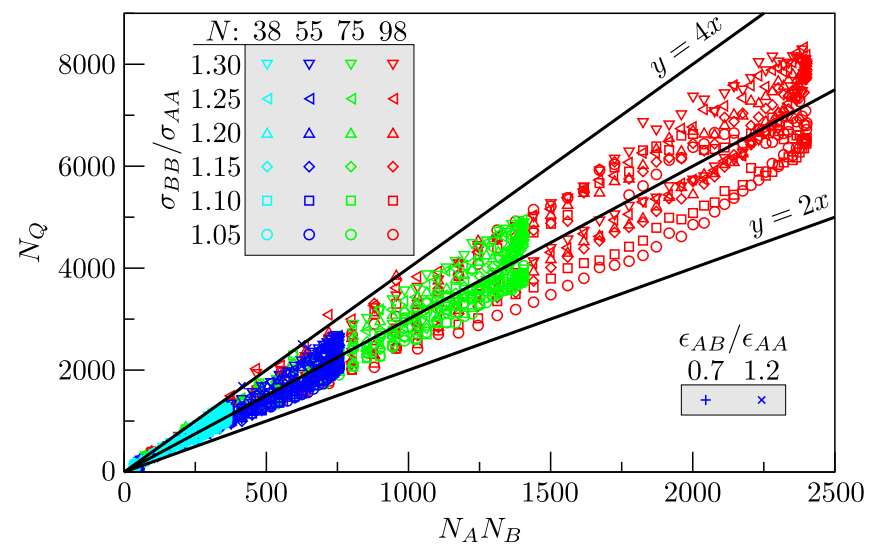

FIG. 4. Mean number of quenches required for convergence to a biminimum.

quench-assisted permutations, which can lead to interesting behaviour. The sublandscape will be single valued if the quench-induced change in $\mathbf{X}$ is reversible for all permutations, in which case the number of distinct isomers will be bounded above by the appropriate combinatorial factor, i.e. $N ! / N_{A} ! N_{B}$ !. Our extensive numerical experiments suggest that $\mathrm{BLJ}_{55}$ closed-shell icosahedra with $\sigma_{B B} / \sigma_{A A} \leq 1.1$ have a single-valued effective sublandscape for all $N_{A}$, and we counted biminima for a few representative cases in this tractable regime.

The results are presented in Fig. 3, showing that many randomly-generated initial homotops are mapped onto a significantly smaller number of distinct biminima. Recall that the mapping itself also scans many homotops (e.g. for $N_{A}=28$, Fig. 3 a accounts for about $3.5 \times 10^{3}$ distinct initial isomers, whereas the total number of unique isomers encountered during the search is around $\left.4 \times 10^{5}\right)$. Note that, although $N ! /\left(N_{A} ! N_{B} !\right)$ peaks at $N_{A}=27$ and 28 , the number of biminima peaks at a higher value of $N_{A}$. This asymmetry is due to smaller (A-type) atoms concentrating in the core of the cluster, reflecting the importance of the free surface.

From Figs. 3a-b we infer that increasing size mismatch raises the number of biminima, but the number remains relatively small. It rises more when the mismatch is increased to $15 \%$ (not shown), but then the closed-shell icosahedral motif is not always preserved, and so the counts are not directly comparable. The number of biminima can be tuned by also adjusting the energy-well parameters in (1). For instance, consider the system with $10 \%$ size mismatch and $\epsilon_{B B} / \epsilon_{A A}=0.5$, in which case the larger species are also less cohesive. We can then set the value of $\epsilon_{A B} / \epsilon_{A A}$ to either 0.7, to encourage segregation, or 1.2 to encourage mixing. These choices show that the number of biminima increases with the mixing preference (see Figs. 3c-d).

To assess our particular method of locating biminima, we now consider the average number of quenches $\left(N_{Q}\right)$

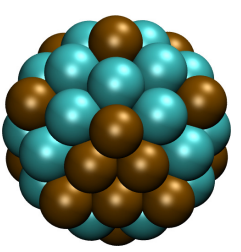

(a)

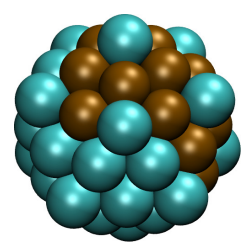

(b)

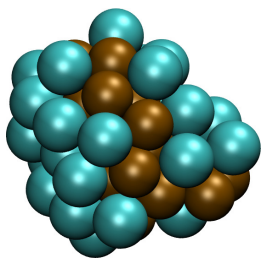

(c)
FIG. 5. Biminima for BLJ $\mathrm{J}_{55}$ with $N_{A}=28$ and $\sigma_{B B} / \sigma_{A A}=$ 1.05 (a), 1.1 (b) and 1.2 (c). A-type atoms are brown (darker) and $B$-type are cyan (lighter).

it takes to converge for a wide range of $\mathrm{BLJ}_{N}$ clusters. Fig. 4 shows that $N_{Q}$ grows linearly with $N_{A} N_{B}$ in all cases, and from our earlier considerations we expect the same trend to hold with respect to $\frac{1}{2} \sum_{\alpha=1}^{M} \sum_{\beta \neq \alpha} N_{\alpha} N_{\beta}$ for a general $M$-component system. The slope of the overall trend is $3 \pm 1$, and there is little variance with $N$, geometry or BLJ parameters. We also observe that, when particle-size mismatch is high $\left(\sigma_{B B} / \sigma_{A A} \gtrsim 1.15\right)$, quench-assisted swaps often lead to significant geometric distortion (see Fig. 5c), but this behaviour does not seem to cause any striking departure from the trend in Fig. 4. Randomly perturbing the coordinates (as well as permuting the labels) to escape from biminima also had no significant effect on the convergence, indicating that the trend may be independent of the sampling approach, which can include the use of elaborate geometric moves $[8,16]$, the Metropolis accept/reject criterion $[6,7]$, or heuristics based on principles of evolution $[9,10]$.

Finally, we illustrate how sampling biminima can facilitate structure prediction. Recall from Fig. 1 that the energy spacing between low-lying homotops may be very small, but the corresponding differences in structure can be significant, with potentially important consequences. For instance, visual inspection of all the biminima in Fig. 1a reveals that $B$-type atoms populate the outer shell in a very specific manner (see Fig. 5b), always occupying all of the twelve vertices and completely filling six/seven facets. This striking pattern is not fully developed in the two lowest configurations marked in Fig. 1c, where at least one of the vertices is occupied by an $A$ type atom. For a nanoalloy with catalytic sites at the vertices, failure to find the lowest-energy mixing pattern could lead to an incorrect conclusion about the availability of desirable sites. For the same system but with half the size mismatch (now 5\%) we find just one biminimum, with the surface mixing pattern reversed: type- $A$ atoms occupy all of the vertex sites and fill just one triangular facet (see Fig. 5a). In addition, despite the obvious differences in the surface mixing patterns, the structures in Figs. 5a-b happen to have the same point-group symmetry $(\mathrm{T})$.

Summary. We define biminima on the potential en- 
ergy surface of a multicomponent system, and we provide a rigorous procedure for locating these special points. We show that sampling the biminima using a random walk can be effective in optimising the structure of binary atomic clusters. This approach is expected to be equally efficient for general multicomponent materials, modelled using any level of theory.

This work was financially supported by EPSRC grant EP/J010847/1 and the ERC. We thank Dr. Chris Forman for coining the term biminimum. D.S. is also grateful to James Farrell and Dr. Jacob Stevenson for useful discussions related to this work.

* Dmitri.Schebarchov@gmail.com

† dw34@cam.ac.uk

[1] R. Ferrando, J. Jellinek, and R. L. Johnston, Chem. Rev. 108, 845 (2008); D. Alloyeau, C. Mottet, and C. Ricolleau, eds., Nanoalloys: Synthesis, Structure and Properties (Springer, 2012); F. Calvo, ed., Nanoalloys: from fundamentals to emergent applications (Elsevier, 2013).

[2] J. Maddox, Nature 335, 201 (1988); S. M. Woodley and R. Catlow, Nature Mater. 7, 937 (2008).

[3] R. J. Saykally and D. J. Wales, Science 336, 814 (2012); A. Hermann, N. W. Ashcroft, and R. Hoffmann, Proc. Natl. Acad. Sci. USA 109, 745 (2012).

[4] S. C. Glotzer and M. J. Solomon, Nature Mater. 6, 557 (2007); D. Frenkel and D. J. Wales, ibid. 10, 410 (2011).

[5] D. J. Wales, Energy landscapes (Cambridge University Press, 2003).
[6] Z. Li and H. A. Scheraga, Proc. Natl. Acad. Sci. USA 84, 6611 (1987); D. J. Wales and J. P. K. Doye, J. Phys. Chem. A 101, 5111 (1997).

[7] S. Goedecker, J. Chem. Phys. 120, 9911 (2004).

[8] G. G. Rondina and J. L. F. Da Silva, J. Chem. Inf. Model. 53, 2282 (2013).

[9] G. M. Morris, D. S. Goodsell, R. S. Halliday, R. Huey, W. E. Hart, R. K. Belew, and A. J. Olson, J. Comput. Chem 19, 1639 (1998).

[10] R. L. Johnston, Dalton Trans. 22, 4193 (2003).

[11] A. R. Oganov and C. W. Glass, J. Chem. Phys. 124, 244704 (2006); Y. Wang, J. Lv, L. Zhu, and Y. Ma, Phys. Rev. B 82, 094116 (2010); A. L.-S. Chua, N. A. Benedek, L. Chen, M. W. Finnis, and A. P. Sutton, Nature Mater. 9, 418 (2010); C. J. Pickard and R. J. Needs, J. Phys.: Condens. Matter 23, 053201 (2011).

[12] J. Jellinek and E. Krissinel, Chem. Phys. Lett. 258, 283 (1996).

[13] R. Ferrando, A. Fortunelli, and R. L. Johnston, Phys. Chem. Chem. Phys. 10, 640 (2008).

[14] J. P. K. Doye and D. J. Wales, Phys. Rev. Lett. 80, 1357 (1998); J. P. K. Doye, D. J. Wales, and M. A. Miller, J. Chem. Phys. 109, 8143 (1998).

[15] M. Sicher, S. Mohr, and S. Goedecker, J. Chem. Phys. 134, 044106 (2011).

[16] T. Ye, R. Xu, and W. Huang, J. Chem. Inf. Model. 51, 572 (2011); X. Lai, R. Xu, and W. Huang, J. Chem. Phys. 135, 164109 (2011).

[17] D. Schebarchov and D. J. Wales, J. Chem. Phys. 139, 221101 (2013).

[18] B. W. Kernighan and S. Lin, Bell Syst. Tech. J. 49, 291 (1970); C. Fiduccia and R. M. Mattheyses, Proc. Nineteenth Design Automation Conf. , 175 (1982).

[19] J. P. K. Doye and L. Meyer, Phys. Rev. Lett. 95, 063401 (2005). 